\title{
IN BRIEF
}

\section{METASTASIS}

The tumour microenvironment drives metastasis

Mlecnik et al. analysed the genetics, levels of genomic instability, vascularization and the immunoscore (a measure of the "immune contexture' of the tumour microenvironment) in a cohort of colorectal cancers. They found that FBXW7 mutations were inversely correlated with metastasis; genomic instability did not correlate with metastasis. However, decreased lymphatic vascularity and reduced immune cytotoxicity were strongly associated with metastasis, suggesting that the tumour microenvironment has a dominant role in metastasis.

ORIGINAL ARTICLE Mlecnik, B. et al. The tumor microenvironment and immunoscore are critical determinants of dissemination to distant metastasis. Sci. Transl Med. 8, 327 ra26 (2016)

\section{$\Rightarrow$ TUMOUR SUPPRESSION}

To EMT or not to EMT?

Transforming growth factor- $\beta$ (TGF $\beta$ ) signalling can be oncogenic or tumour suppressive. Because SMAD4, a mediator of TGF $\beta$ signalling, is frequently inactivated in pancreatic ductal adenocarcinoma (PDAC), David et al. used this cancer type to investigate the dichotomous role of TGF $\beta$ in tumorigenesis. In PDAC cells without SMAD4 inactivation, TGF $\beta$ induced epithelial-mesenchymal transition (EMT), but rather than promoting tumorigenesis this induced cell death. Further investigation revealed that TGF $\beta-S M A D 4$ remodelled the expression of many transcription factors, including upregulating SOX4 and repressing the expression of KLF5, which prevents SOX4-induced apoptosis. Thus, TGF $\beta$ signalling is tumour suppressive by inducing SOX4-mediated apoptosis in PDAC.

ORIGINAL ARTICLE David, C. J. et al. TGF- $\beta$ tumor suppression through a lethal EMT. Cell 164, 1015-1030 (2016)

\section{$\Rightarrow$ EXERCISE}

\section{Running mobilizes tumour cell killers}

There is accumulating evidence that exercise can suppress tumorigenesis in humans and improve outcomes in patients with cancer but the mechanisms are unclear. Using five mouse models of cancer, Pedersen et al. showed that voluntary running reduced tumorigenesis and also reduced tumour progression by $\sim 60 \%$. These reductions were associated with increased interleukin-6 (IL-6)-sensitive natural killer (NK) cell infiltration of tumours and these cells were mobilized by running-induced epinephrine, This study raises many questions. In particular, as mice are naturally very active it is interesting to debate whether this demonstrates that increased exercise suppresses tumorigenesis and tumour progression or that increased sedentary behaviour promotes them.

ORIGINAL ARTICLE Pedersen, L. et al. Voluntary running suppresses tumor growth through epinephrine- and IL-6-dependent NK cell mobilization and redistribution. Cell Metab. 23, 554-562 (2016)

\section{GENETICS}

\section{Pushing boundaries}

Chromosome structures called insulated neighbourhoods allow the control of genes within that structure, and Hnisz, Weintraub et al. investigated whether proto-oncogenes might also be affected by this regulation. They found that recurrent deletions that ablate the boundaries of insulated neighbourhoods occur in many types of cancer, indicating that their disruption may be common in tumorigenesis.

ORIGINAL ARTICLE Hnisz, D. et al. Activation of proto-oncogenes by disruption of chromosome neighborhoods. Science http://dx.doi.org/10.1126/science.aad9024 (2016) 